\title{
SOME PROPERTIES OF CHARACTERS OF FINITE SOLVABLE GROUPS
}

\author{
BY PAUL FONG \\ Communicated by Richard Brauer, October 31, 1959
}

The purpose of this note is to announce two results on properties of the characters of a finite solvable group $G$. The first is a necessary and sufficient condition on the irreducible characters of $G$ in order that its Sylow subgroups be abelian. The second is a necessary condition on the irreducible characters of $G$ in order that certain $p$-subgroups of $G$ be abelian. These results confirm certain assertions in a conjecture by Brauer in [1]. In order to formulate the results, some definitions from modular representation theory are needed. For the motivation behind these definitions, see [2].

Let $G$ be a finite group of order $g, F$ an algebraic number field of finite degree such that the irreducible representations of $G$ in $F$ are absolutely irreducible. If $p$ is a fixed rational prime, there is a grouping of the absolutely irreducible characters $\chi_{1}, \chi_{2}, \cdots, \chi_{k}$ of $G$ into disjoint sets called the blocks of $G$ (for the prime $p$ ). This grouping can be made in the following way: let $\mathfrak{p}$ be a prime ideal divisor of $p$ in $F$. Then two irreducible characters $\chi_{i}$ and $\chi_{j}$ are in the same block $B_{t}$ of $G$ if and only if

$$
\frac{g}{n(\sigma)} \frac{\chi_{i}(\sigma)}{x_{i}} \equiv \frac{g}{n(\sigma)} \frac{\chi_{j}(\sigma)}{x_{j}}(\bmod b)
$$

for all $\sigma$ in $G$. Here $n(\sigma)$ is the order of the normalizer of $\sigma$ in $G, x_{i}$ the degree of $\chi_{i}, x_{j}$ the degree of $\chi_{j}$. Since the numbers occurring in the above congruence can be computed from the character table of $G$, the blocks of $G$ can therefore be determined once the character table of $G$ is known.

THEOREM 1. Let $G$ be a finite solvable group, $B_{1}$ the block containing the principal or 1-character of $G$. Then a necessary and sufficient condition for the Sylow p-subgroups of $G$ to be abelian is that every character in $B_{1}$ has degree relatively prime to $p$.

Since our two results are related, we shall state the second result before indicating their proofs. Let $p^{a}$ be the highest power of $p$ dividing the order of the finite group $G$ ( $G$ not necessarily solvable). The defect of a block $B_{t}$ of $G$ is the smallest non-negative integer $d$ such that $p^{a-d}$ divides the degree $x_{i}$ of every character $\chi_{i}$ in $B_{t}$. If the exact power of $p$ dividing the degree of a character $\chi_{i}$ in $B_{t}$ is $p^{a-d+e}$, where $e \geqq 0$, we define the height of $\chi_{i}$ to be $e$. In [2] there is associated to 
each block $B_{t}$ its defect group $D$, a $p$-subgroup of $G$ determined uniquely up to conjugate subgroups in $G$. $D$ has order $p^{d}$, where $d$ is the defect of $B_{t}$. The conjecture by Brauer is that the defect group $D$ of a block $B_{t}$ is abelian if and only if every character in $B_{t}$ has height 0 . Theorem 1 is a special case of the conjecture, since the defect groups of $B_{1}$ are the Sylow $p$-subgroups of $G$.

THEOREM 2. Let $G$ be a finite solvable group, $B_{t}$ a block of $G$ with $D$ as defect group. If the center of $D$ has index $p^{c}$ in $D$, then every character in $B_{t}$ has height less than or equal to $c$. In particular, if $D$ is abelian, then every character in $B_{t}$ has height 0.

The necessity of the condition in Theorem 1 is included in Theorem 2. The proofs of both theorems use induction on the order of $G$. In order to carry through the induction, the following lemma is needed: let $H$ be a normal subgroup of prime index of a group $G, G$ not necessarily solvable. If $\chi_{i}$ is an irreducible character of $G$ in a block with $D$ as defect group, then some irreducible constituent of the restriction $\chi_{i} \mid H$ of $\chi_{i}$ to $H$ is in a block of $H$ with $D \cap H$ as defect group. Here $D \cap H \neq D$ only in the case $(G: H)=p$ and the irreducible constituents of $\chi_{i} \mid H$ are in one block of $H$. Theorem 2 follows by a direct application of this lemma except in the case where $D \cap H \neq D$ and $\chi_{i} \mid H$ is reducible. However, it is then possible to show that the center of $D$ lies in $H$ and induction will work.

The proof of the sufficiency of the condition in Theorem 1 is rather lengthy. The bare outline of the proof is as follows. By induction we can reduce the problem first to the case where every maximal normal subgroup of $G$ has index $p$, secondly to the case where every minimal normal subgroup of $G$ has order a $p$-power. These restrictions on the maximal and minimal normal subgroups of $G$ imply that the minimal normal subgroups of $G$ have order $p$. If Theorem 1 were not true, it would then be possible to construct an irreducible character of a suitable subgroup $M$ of $G$ for which Theorem 2 would be false. The subgroup $M$ is the normalizer in $G$ of a fixed Sylow $p$-subgroup of $H$, where $H$ is a maximal normal subgroup of $G$.

\section{REFERENCES}

1. R. Brauer, Number theoretical investigations on groups of finite order, Proceedings of the International Symposium on Algebraic Number Theory, Tokyo, 1956, pp. 5562.

2. Zur Darstellungstheorie der Gruppen endlicher Ordnung, Math. Z. vol. 63 (1956) pp. 406-444.

HARVARD UNIVERSITY 